\title{
CYCLICAL CORRELATIONS, CREDIT CONTAGION, AND PORTFOLIO LOSSES
}

\author{
Kay Giesecke \\ Stefan Weber \\ Cornell University \\ TU Berlin
}

January $17,2003^{*}$

\begin{abstract}
We model aggregate credit losses on large portfolios of financial positions contracted with firms subject to both cyclical default correlation and direct default contagion processes. Cyclical correlation is due to the dependence of firms on common (macro-) economic factors; credit contagion phenomena are associated with the local interaction of firms with their business partners. We provide an explicit normal approximation of the distribution of total portfolio losses, which is the key to the measurement and management of aggregated credit loss risk. Based on this result we quantify the relation between the variability of global economic fundamentals, strength of local interaction between firms, and the fluctuation of portfolio losses. In particular, we find that cyclical oscillations in fundamentals dominate average portfolio losses, while local firm interaction and the associated contagion processes cause additional fluctuations of losses around their average. The strength of the contagion-induced loss variability and hence the degree of extreme loss risk depends on the complexity of the business partner network, a relation that was recently confirmed by empirical studies.
\end{abstract}

Key words: cyclical correlation, credit contagion, portfolio losses, voter model, Bernoulli mixture model

\footnotetext{
*Giesecke is with the Department of Operations Research and Industrial Engineering, Cornell University, Ithaca, NY 14853-3801, USA, Phone (607) 255 9140, Fax (607) 255 9129, email: giesecke@orie.cornell.edu. Weber is with the Department of Mathematics, TU Berlin, Sekretariat MA 7-4, Str. des 17. Juni 136, 10623 Berlin, Germany, Phone +49 303142 7601, Fax +49 303142 1695, email weber@math.tu-berlin.de. Giesecke acknowledges financial support by Deutsche Forschungsgemeinschaft. Weber acknowledges financial support by Deutsche Forschungsgemeinschaft via Graduiertenkolleg 'Stochastische Prozesse und Probabilistische Analysis'.
} 


\section{Introduction}

One of the long-lasting discussions in economics concerns the explanation of aggregate economic activity. In this paper we contribute to this discussion by studying the fluctuation of aggregate credit losses on large portfolios of financial positions. Our explicit results provide a number of significant insights relevant to both risk measurement and management in financial institutions and supervisory authorities.

Default rates of firms and hence credit losses vary substantially even on a high level of aggregation [see, in particular, the regular studies of the various credit rating agencies, for example Keenan (2000)]. One potential explanation is easily conceived: firms' ability to generate cash flows and hence their default proneness fluctuates with the fundamentals of the economy, such as specific factor prices, demand for manufactured goods, or production costs. The dependence of firms' on the general (macro-) economic environment induces dependence between firms' defaults. A high degree of such positive cyclical default correlation and thus a high fluctuation in aggregate default rates and credit losses would result from an economy-wide synchronized variation in fundamental variables, which is simultaneously disastrous for a large number of firms.

In this article we emphasize the importance of the economy's microeconomic structure and argue that the effect of variations in fundamental macroeconomic variables can amplify with the appearance of direct connections between firms. These direct inter-firm links are typically associated with borrowing and lending contracts or other legally binding relationships, such as parent-subsidiary structures; they provide a channel for the direct contagion of economic distress from one firm to other firms. A characteristic example are interbank lending agreements, which refer to banks' mutual claims. Provided that these claims are neither collateralized nor insured against, the financial distress of one institution, triggered by management failure or adverse fundamentals, may spread to several other institutions in the lending chain through default on due obligations. Such bank-contagion effects are widely discussed in the micro-economic literature, for example Allen \& Gale (2000); empirical evidence is presented in Upper \& Worms (2002) and Schoenmaker (2000). A similar contagion mechanism is also associated with non-financial firms through the institution of trade credit, which link suppliers and buyers of goods through a chain of obligations. For a micro-economic model see, for example, Kiyotaki \& Moore (1997). Legal networks as those related to parent-subsidiary struc-

tures are prone to contagion effects as well. Empirical evidence supporting the 
existence of contagion effects between non-financial firms is presented by Lang \& Stulz (1992).

With the existence of credit contagion phenomena a change in economic fundamentals has two separate effects on an individual firm's credit-worthiness, i.e. its ability to generate cash flows and to honor its obligations. First, it acts upon the firm directly. Second, there is an indirect effect due to the simultaneous influence on the financial health of associated business partners, which might jeopardize or contribute to the fulfilment of existing obligations. The effect of a change in fundamentals can thus amplify, basically in both positive and negative direction. Consider some fixed part of the economy and suppose there is an adverse change in the macro-environment. In the first place, this deterioration may affect only a limited number of firms (due to their specific technology, for example). However, the distress can propagate through the business partner network and eventually affect a larger number of firms than those initially concerned. This corresponds to an increased degree of (adverse) fluctuation in default rates and associated credit losses relative to the fluctuation due to the variability of macro-economic fundamentals. The existence of such additional contagion-induced oscillations in default rates has been empirically confirmed by Schoenmaker (2000), for example.

Excessive adverse fluctuation of default rates is typically connected with the economic distress of lending institutions, and therefore endangers the stability of the financial system. Besides being of critical importance for individual banks' risk management, the design of effective supervising policies and intervention strategies calls for a thorough understanding of the variability of aggregate losses. In this paper we study the typical and excessive fluctuation of aggregate credit losses on large portfolios of financial positions, taking into account both cyclical default correlation and credit contagion processes. From a methodological point of view, this provides a reconciliation of the cyclical correlation-based class of Bernoulli mixture models [Frey \& McNeil (2001)] and a pure credit contagion based approach, which was recently introduced by Giesecke \& Weber (2002).

Bernoulli mixture models have become a standard for the measurement and management of credit loss risk in financial institutions. Examples include the models put forward by KMV [Kealhofer (1998)], J.P. Morgan [Gupton, Finger \& Bhatia (1997)], Credit Suisse Financial Products [CSFP (1997)], and McKinsey [Wilson (1997)]. For an overview of these models we refer to Crouhy, Galai \& Mark (2000). In this class of models the fluctuation of credit losses is due to variation of economic fundamentals only, so that firms' interdependence is related to cyclical correlation effects only. As we have argued above and as 
suggested by recent empirical investigations [Schoenmaker (2000), in particular], such an approach typically underestimates the degree of loss fluctuation to be expected. A pure contagion based approach, on the other hand, does typically not explicitly account for the variation of fundamentals, but focuses instead on the credit loss fluctuation due the local interaction of firms with their business partners. In this paper we connect these two conceptionally different frameworks. In particular we will enlarge the Bernoulli mixture class with models accommodating both global cyclical and local contagion effects.

In our model firms interact with their business partners in a lattice-type economy. With each firm we associate a binary state variable, which designates the firm's liquidity state with respect to the direct interaction with its business partners. Specifically, a firm's liquidity account can be severely "stressed" if business partners fail to honor due obligations. In this case the firm may not be able to generate sufficient cash flow to invest in production opportunities and to honor its own obligations. If the firm can buffer the adverse effects from defaulting business partners (through sufficient reserves, for example), then its liquidity state is considered "stable". We suppose that the migration of a firm from one liquidity state to another is a Poisson event with an intensity being proportional to the number of business partners in opposite state. The idea here is that if a stable firm's partners default on obligations, then the probability of this firm becoming liquidity stressed as well increases with the number of failing partners, since at some point the available liquidity reserves will be exhausted. Vice versa, the probability of a liquidity stressed firm to overcome the shortage increases with the number of financially healthy partners which honor due obligations timely.

The continuous-time Markov process describing the joint evolution of firms' interaction-induced liquidity state converges as time approaches infinity. For this steady equilibrium state we describe the associated state of the macroeconomy (the business environment) by a random vector with given distribution. We can then turn to the losses a financial institution might incur from holding financial positions with firms in the interaction-based economy. Such positions might include straight loans, bonds, and other debt instruments, as well as derivatives whose counterparties are subject to credit deterioration. In our model the credit losses on positions contracted with a particular firm is a random variable, whose distribution depends on both the macro-environment and the firm's individual liquidity state resulting from the local interaction with its business partners. This allows to accommodate the global macro-economic factors and the local contagion processes in explaining aggregate credit loss fluctuations. 
Our main result consists of an explicit normal approximation of the distribution of aggregate losses on a large portfolio of positions, whose issuers are subject to the macro-economic environment and credit contagion processes. This approximation is the key to the measurement and management of the portfolio's aggregated credit loss risk.

In order to analyze the fluctuations of aggregate portfolio losses in comparison with those implied by Bernoulli mixture models, we provide a Bernoulli mixture type representation of our model and thereby enlarge the existing Bernoulli mixture model class. With a view towards implementation, for this representation of our model it is possible to exploit the estimation techniques employed by the Bernoulli mixture models currently in use in the financial industry. We propose an estimation strategy for the contagion parameters of our model as well.

We find that average losses on large portfolios of financial positions are dominated by cyclical oscillations in the economy's fundamentals. Local firm interaction and the associated contagion processes lead to additional fluctuations of losses around their averages. This means that the existence of contagion processes corresponds to the presence of additional loss risks on the portfolio level, which cannot be attributed to the variability in the macro-economy. The strength of the additional contagion-induced loss variability and hence the degree of extreme loss risk depends on the complexity of the business partner network, i.e. the degree of connectedness between firms. Specifically, the loss variability increases and the tail of the loss distribution becomes fatter with decreasing complexity of business partner relations. This finding was recently confirmed empirically in the banking sector by Upper \& Worms (2002).

The balance of this paper is organized as follows. In Section 2 we define an economy where firms interact with each other within a business partner network specified by a multi-dimensional lattice. We postulate contagion dynamics and analyze the long-run behavior of firms' interaction-induced state. In Section 3 we examine credit losses due to macro-economic fluctuations as well as contagion effects. We specifically provide a normal approximation to the distribution of aggregate losses. A Bernoulli mixture type representation of our model is provided in Section 4, where we also examine the typical and extreme fluctuation of aggregate losses. An estimation strategy for the parameters is discussed in Section 5. Section 6 concludes and discusses some important implications of our results for the regulation of financial institutions and the control of systemic risk. 


\section{Economy and Firms}

Our economy consists of a collection $F$ of firms. An arbitrary firm $i \in F$ interacts with a collection $\mathcal{N}(i) \subseteq F \backslash\{i\}$ of business partners, or neighbors. Typical business partners include suppliers of goods in the manufacturing process and buyers of manufactured products. The firm's creditors, such as suppliers in trade credits, banks, shareholders, or investors in the firm's public debt, as well as its borrowers (think of customers which are granted a trade credit), can also be considered as business partners. For simplicity, we assume that a firm's interaction with its neighbors is symmetric, in the sense that

$$
j \in \mathcal{N}(i) \Rightarrow i \in \mathcal{N}(j) .
$$

If we connect all firms $i \in F$ with their neighbors $j \in \mathcal{N}(i)$, we get an undirected graph which characterizes the business relations of the firms. For tractability, however, we shall assume a simple neighborhood structure and identify firms with their location on the $d$-dimensional integer lattice $F=\mathbb{Z}^{d}$. On this lattice we define the neighborhood $\mathcal{N}(i)$ of a firm $i$ by

$$
\mathcal{N}(i)=\{j:|j-i|=1\},
$$

where $|\cdot|$ denotes the length of the shortest path between two firms on the lattice. The dimension $d$ of the lattice can be interpreted as the degree of complexity of the business partner network. With increasing $d$ the structure of inter-firm connections becomes more complex. The larger $d$, the more business partners has any individual firm. At the same time the number of indirect inter-firm links of given length increases.

Having defined the structure of our interaction-based economy, let us now consider the process of interaction in more detail. In the business partner network firms are linked through a chain of obligations. If one link in this chain does not honor obligations timely or defaults entirely, then this immediately reduces the amount of liquidity available to this firm's business partners. We can here think, for example, of a temporary liquidity shortage leading to a payment default of a buyer in a trade credit in the first place. Supposing that the immediate liquidation value of the underlying goods used as collateral is sufficiently low, then this default may eventually result in the supplier becoming short of liquidity as well. Given the lack of liquidity, the supplier may be prevented from investing in production opportunities and realizing the associated returns in the future. This reduction in the supplier's cash flow ability may lead to the supplier defaulting on obligations with other business partners, resulting in a 'chain reaction' within the credit trade network. We will refer to this cascading behavior as credit contagion. 
We are now going to establish a simple probabilistic model for the time dynamics of credit contagion. To this end, let us associate with each firm $i \in \mathbb{Z}^{d}$ a state variable $\xi(i) \in\{0,1\}$, which designates the firm's liquidity state with respect to the interaction with its business partners $\mathcal{N}(i)$. State $\xi(i)=1$ means that firm $i$ 's liquidity reserves are stressed and might be insufficient to honor due obligations. State $\xi(i)=0$ means that firm $i$ is financially healthy and honors its obligations to business partners timely.

We postulate that a transition of firm $i$ from state $\xi(i)$ to state $1-\xi(i)$ is a Poisson event, whose intensity is proportional to the number $\mid\{j \in \mathcal{N}(i)$ : $\xi(j)=1-\xi(i)\} \mid$ of neighboring firms in opposite state. This corresponds to the idea that if a currently stable firm's business partners default on obligations, then the probability of this firm becoming liquidity stressed as well increases with the number of failing partners, since at some point the available liquidity reserves will be exhausted. Vice versa, the probability of a liquidity stressed firm to overcome the shortage increases with the number of financially healthy business partners which honor due obligations timely. Specifically, we assume that the evolution of firms' liquidity state over time is modeled by a continuoustime Feller process $\left(\eta_{t}\right)_{t \geq 0}$ with state space $X=\{0,1\}^{\mathbb{Z}^{d}}$ and transition rate $c$ given by

$$
c(i, \xi)=\left\{\begin{array}{rlll}
\frac{1}{2 d} \sum_{j \in \mathcal{N}(i)} \xi(j) & \text { if } & \xi(i)=0 \\
\frac{1}{2 d} \sum_{j \in \mathcal{N}(i)}[1-\xi(j)] & \text { if } & \xi(i)=1
\end{array} .\right.
$$

This is known as the voter model in the theory of interacting particle systems [Liggett (1985) and Liggett (1999)].

Having defined the contagion dynamics, we are now interested in the asymptotic behavior of $\eta_{t}$ as $t \rightarrow \infty$ and the equilibrium distributions of firms' liquidity state. Throughout, we let $\mu$ denote the initial distribution of $\eta$, which we may think of arising from general (macro-) economic conditions. We assume that $\mu$ is translation-invariant and denote by

$$
\rho=\mu\{\xi: \xi(i)=1\}
$$

the probability under $\mu$ that an arbitrary firm $i$ is liquidity stressed. In particular, the translation-invariance of $\mu$ implies that the firms in our economy are homogeneous with respect to $\rho$. While $\rho$ is invariant under the contagion dynamics, the economy can change drastically on the macroscopic level. The structure of the equilibrium distributions depends on the complexity of the business partner network. 
For a simple connectivity structure with $d=1,2$, the process $\eta$ clusters: in the long run $t \rightarrow \infty$ the economy ends up in one of two possible extreme scenarios. Asymptotically, with probability $\rho$ all firms are liquidity stressed, and with probability $1-\rho$ all firms are stable. Let us suppose that initially $\rho$ is high, so that the probability that an individual firm is liquidity stressed is high. Then random clusters of liquidity stressed firms emerge with high probability, while clusters of stable firms emerge only with low probability. The size of the clusters changes through random fluctuations, but some of the clusters merge and form large growing clusters. In the long run the entire economy is a single cluster of firms of the same type. Since $\rho$ is large, the probability that all firms are finally liquidity-stressed is high.

The limiting behavior of $\eta$ differs for higher dimensions $d>2$. Here in the long run the process $\eta$ coexists, meaning that heterogeneity in firms' states will appear for $\rho \in(0,1)$. Random clusters of firms of equal state appear here only locally; they do not persist and grow not in the same way we observed with $d=1,2$. The equilibrium distribution of $\eta_{t}$ for $t \rightarrow \infty$ is given by the mixture

$$
\int_{[0,1]} \nu_{\rho} Q(d \rho)
$$

cf. Giesecke \& Weber (2002). Here, $\nu_{\rho}$ is the extremal invariant measure of the basic voter model in dimension $d>2$ with parameter $\rho \in[0,1]$ [cf. Liggett (1999)], and $Q$ is the distribution of the empirical average of liquidity-stressed firms 1 in the whole economy:

$$
\lim _{n \rightarrow \infty}\left|\Lambda_{n}\right|^{-1} \sum_{i \in \Lambda_{n}} \xi(i)=\bar{\rho}
$$

where $\Lambda_{n}=[-n, n]^{d}$. In general, $\bar{\rho}$ is not deterministic, but random. The empirical average of the number $\bar{\rho}$ of firms in state 1 and its distribution $Q$ are invariant under the contagion dynamics. Nevertheless, interaction between firms strongly effects the correlation between the states of different firms. For any finite number of firms, the probability to find many firms in the same state is higher than in the case of independent firms; the equilibrium state distribution exhibits heavy tails.

\section{Portfolio Losses}

In this section we examine the fluctuation of aggregate losses on a portfolio of debt contracts written by firms subject to the contagion processes described in the previous section. Throughout, we suppose that the economy is in a steady 
equilibrium state, in the sense that the distribution of firms' interaction based state is invariant. The steady state distribution of the liquidity configuration will be denoted by

$$
\mu=\int_{[0,1]} \nu_{\rho} Q(d \rho)
$$

We consider a financial institution holding a portfolio $\Lambda_{n}=[-n, n]^{d}$ of financial positions contracted with the firms in the interaction-prone economy. The parameter $n \in \mathbb{N}$ determines the portfolio's size, i.e. the number $\left|\Lambda_{n}\right|=$ $(2 n+1)^{d}$ of firms in the portfolio. The market value of a portfolio position is subject to the credit quality, or ability to generate future cash flows, of its issuer or counterparty. Such positions can include not only loans, bonds, or other debt instruments, but also derivatives written by default-prone counterparties. Due to adverse changes in a counterparty's credit quality the market valuation of the corresponding positions can be severely reduced. Risk measurement aims at evaluating the potential losses induced by credit quality deterioration of firms in portfolio $\Lambda_{n}$. Denoting the losses on positions contracted with firm $i \in \Lambda_{n}$ by the random variable $U(i)$, we are thus interested in the distribution of aggregated portfolio losses

$$
L_{n}=\sum_{i \in \Lambda_{n}} U(i)
$$

In the subsequent Section 3.1 we model the probabilistic properties of the position losses, which then allows us in Section 3.2 to study the distribution of aggregate losses $L_{n}$ in detail.

\subsection{Position Losses}

The loss $U(i)$ the financial institution incurs from positions contracted with firm $i \in \Lambda_{n}$ will depend on the credit quality of the firm, i.e. its ability to generate the required cash flows in the future. In our model, this cash flow ability is not only determined by the firm's liquidity state resulting from the interaction with its business partners, but also by the state of the general macro-economy (the business environment) in which the firms operate. In this sense both "global" business cycle fluctuations and "local" interaction-induced contagion processes corresponding to the economy's micro firm structure affect credit losses. This is in fact an important conceptual advancement over the existing Bernoulli mixture models [Frey \& McNeil (2001)], which have become a standard for credit risk measurement and management in financial institutions. The key assumption of Bernoulli mixture models is that credit losses 
are conditionally independent given the macro-economic state. Dependence between losses on firms' positions arises through the dependence of firms on the common macro-state variables. Here firms' interdependence is related to cyclical correlation effects only; effects stemming from direct firm interaction are not captured. Our model, in contrast, captures both cyclical and contagion effects.

For the specification of the probabilistic properties of the position losses $U(i)$ we note first that for the credit contagion process being in some tuned steady equilibrium state, we know the joint distribution (6) of firms' interactioninduced liquidity state. The state of the general (macro-) economy prevailing in the steady state will then described by some random vector $K \in \mathbb{R}^{p}$ with given distribution $\kappa$. The vector $K$ is common to all firms and captures the economy's business environment. Elements of $K$ may include indicators of the stage of the business cycle, commodity and factor prices, inflation rates, or interest rates. We can think of $K$ as systematic factors which may affect different firms to a different degree. For example, a highly levered firm is more affected in a high interest rate environment then a firm with low debt load.

Now our key assumptions on the probabilistic structure of the $U(i)$ are as follows. Conditional on the macro-economic state $K \in \mathbb{R}^{p}$ and the liquidity profile $\xi \in\{0,1\}^{\mathbb{Z}^{d}}$, losses are independent. The conditional loss distribution of a firm is denoted by $M_{k, x}$; it depends only on the economy-wide macroeconomic state $k$ and the firm-specific liquidity state $x$. For technical reasons, we assume that the mapping

$$
\begin{cases}\mathbb{R}^{p} \times\{0,1\} & \rightarrow \mathcal{M}_{1}\left(\mathbb{R}_{+}\right) \\ (k, x) & \mapsto M_{k, x}\end{cases}
$$

is measurable. Here, $\mathcal{M}_{1}\left(\mathbb{R}_{+}\right)$denotes the space of Borel probability measures on the positive real line with the weak topology. Moreover, we will suppose that all measures $M_{k, x}$ with $(k, x) \in \mathbb{R}^{p} \times\{0,1\}$ are supported in a common interval $[0, c]$ for some $c>0$.

Given $k$ and $x$ we denote the expected losses conditional on these states by

$$
l_{x}(k):=\int u M_{k, x}(d u)
$$

and assume that for every $k \in \mathbb{R}^{p}$ the inequality $l_{0}(k)<l_{1}(k)$ holds.

\subsection{Distribution of Portfolio Losses}

Having specified the position loss distributions, let us turn to the distribution of portfolio losses. Recall that $\kappa$ is the distribution of the macro-economic 
factor and that $\mu$ given by (6) governs firms' equilibrium liquidity state. In terms of mixtures of the position loss distributions $M_{k, x}$ the firms' joint loss distribution $\beta$ can be written as

$$
\beta(d w)=\iint\left(\otimes_{i \in \mathbb{Z}^{d}} M_{k, \xi(i)}\right)(d w) \mu(d \xi) \kappa(d k), \quad w \in \mathbb{R}_{+}^{\mathbb{Z}^{d}} .
$$

We are interested in the law of aggregated losses $L_{n}$ under the measure $\beta$.

As for average losses in the whole economy, by law of a large numbers

$$
\lim _{n \rightarrow \infty} \frac{L_{n}}{\left|\Lambda_{n}\right|}=\bar{\rho} \cdot l_{1}(K)+(1-\bar{\rho}) \cdot l_{0}(K),
$$

$\beta$-almost surely. The random variables $K$ and $\bar{\rho}$ are independent and distributed according to $\kappa$ and $Q$, respectively. It appears that in the limit all loss fluctuations due to the fluctuation in the conditional position losses $M_{k, x}$ are averaged out; only their expectations $l_{x}(k)$ given the interaction state $x \in\{0,1\}$ and the macro-factor $k \in \mathbb{R}^{p}$ enter average losses.

In order to get a more detailed picture about the fluctuation of losses and their relation to the state of the economy and the interaction between firms, we will now provide a normal approximation of aggregate losses. To this end we introduce the function

$$
\tilde{l}: \begin{cases}\mathbb{R}^{p} & \rightarrow[0,1]^{2} \\ k & \mapsto\left(l_{0}(k), l_{1}(k)\right)\end{cases}
$$

and define

$$
\tilde{\psi}_{d, a}(r ; \rho, k):=\psi_{d, a}(r ; \rho, \tilde{l}(k)),
$$

where, letting $\Phi$ denote the standard normal distribution function,

$$
\psi_{d, a}\left(r ; \rho, l_{0}, l_{1}\right)=\Phi\left(\frac{r^{1 / 2} m\left(\rho, l_{0}, l_{1}\right)-r^{-1 / 2} a}{\left(l_{1}-l_{0}\right) \sigma(d, \rho) r^{1 / d}}\right), \quad a>0, \quad r>0 .
$$

Here

$$
m\left(\rho, l_{0}, l_{1}\right)=\rho \cdot l_{1}+(1-\rho) \cdot l_{0}
$$

and $\sigma^{2}=\sigma^{2}(d, \rho)$ is the constant given by

$$
\sigma^{2}=\rho(1-\rho) \cdot \frac{\gamma_{d} \cdot d}{2^{d+3} \pi^{d / 2}} \cdot \Gamma\left(\frac{d-2}{2}\right) \cdot \int_{[-1,1]^{d}} \int_{[-1,1]^{d}} \frac{1}{\|x-y\|_{2}^{d-2}} d x d y,
$$

where $\Gamma$ is the Gamma-function and $\gamma=\gamma_{d}$ is given by

$$
\frac{1}{\gamma}=(2 \pi)^{-d} \int_{(-\pi, \pi)^{d}}\left(1-\frac{1}{d} \sum_{m=1}^{d} \cos x_{m}\right)^{-1} d x .
$$


Numerical values of $\gamma_{d}$ can be found in Kondo \& Hara (1987) for various dimensions $d$. We are now ready to approximate aggregate losses $L_{n}$. The proof of the theorem is given in the Appendix.

Theorem 3.1. Let $d>2$, and assume that $Q(\{0\})=Q(\{1\})=0$. For a large portfolio, the distribution of the losses $L_{n}$ can uniformly be approximated, i.e. the following inequalities hold:

$$
\begin{aligned}
& \sup _{a \in \mathbb{R}}\left|\beta\left(L_{n} \geq a\right)-\iint \tilde{\psi}_{d, a}\left(\left|\Lambda_{n}\right| ; \rho, k\right) Q(d \rho) \kappa(d k)\right| \\
= & \sup _{a \in \mathbb{R}}\left|\beta\left(L_{n} \geq a\right)-\iint \psi_{d, a}\left(\left|\Lambda_{n}\right| ; \rho, l_{0}, l_{1}\right) Q(d \rho) \kappa^{\tilde{l}}\left(d\left(l_{0}, l_{1}\right)\right)\right| \\
\leq & \epsilon_{n},
\end{aligned}
$$

where $\epsilon_{n} \rightarrow 0$ as $n \rightarrow \infty$. Here, $\kappa^{\tilde{l}}=\kappa \circ \tilde{l}^{-1}$ is the distribution of $\tilde{l}$ under the measure $\kappa$.

For $r>0$ we define the function

$$
\Psi_{d, a}(r)=\iint \psi_{d, a}\left(r ; \rho, l_{0}, l_{1}\right) Q(d \rho) \kappa^{\tilde{l}}\left(d\left(l_{0}, l_{1}\right)\right) .
$$

Heuristically, interpolation between sizes of portfolios implies that $\Psi_{d, a}(r)$ approximates the probability of a loss larger than $a \in \mathbb{R}_{+}$for portfolio size $r>0$ sufficiently large. We observe that the distribution $\kappa$ of the macro-factor $K$ enters the approximation only via the image measure $\kappa^{\tilde{l}}=\kappa \circ \tilde{l}^{-1}$. That is, random fluctuations of the conditional position losses $U(i)$ around their means given the interaction state $\xi(i)$ and the macro-factor $K$ are averaged out in the normal approximation; all that matters are the expected position losses $\tilde{l}=\left(l_{0}, l_{1}\right)$ under the distribution $\kappa$ of the macro-economic factor $K$.

The shape of the approximate loss distribution depends on the size of the contagion parameter $d$. Contagion induces additional fluctuations of the losses in large portfolios around their random means which are determined by the macro-economic factor $K$ and the average number of liquidity-stressed firms $\bar{\rho}$. It will be instructive to re-parameterize the model in terms of the variables $m, \Delta, \rho$ where

$$
\begin{aligned}
m & =\rho \cdot l_{1}+(1-\rho) \cdot l_{0}, \\
\Delta & =l_{1}-l_{0} .
\end{aligned}
$$

The variable $m$ describes the average loss in the whole economy which is almost surely defined by the limit

$$
\lim _{n \rightarrow \infty} \frac{L_{n}}{\left|\Lambda_{n}\right|}=m .
$$


$\Delta$ is given by the difference between the expected losses $l_{1}(k)$ and $l_{0}(k)$.

The distribution $\kappa$ of the macro-economic factor and the distribution $Q$ of the average liquidity-state induce a joint distribution of the triple $(m, \Delta, \rho)$. For simplicity, we assume that $Q$ is a Dirac measure concentrated on $\frac{1}{2}$ and denote the joint image law of $(m, \Delta)$ by $v(d m, d \Delta)$. We remark that the influence of the distribution $Q$ on the contagion effect resembles qualitatively the implications induced by the distribution of $m$ which we will discuss in detail below.

From (11) we obtain the approximate probability of a loss larger than $a \in \mathbb{R}_{+}$for a portfolio of size $r>0$ in terms of the new parameters:

$$
\Psi_{d, a}(r)=\int \Phi\left(\frac{\sqrt{r} \cdot m-a / \sqrt{r}}{\Delta \cdot \sigma(d, \rho) \cdot r^{1 / d}}\right) v(d m, d \Delta)
$$

We will investigate the contagion effect on the shape of the approximate loss distributions for different specifications of the measure $v$, namely the three cases:

(a) $\Delta$ fixed, $m$ random;

(b) $\Delta$ random, $m$ fixed; and

(c) $\Delta, m$ both random.

For each of the choices for the law of $m$ we will compare the contagion effect for degrees of connectedness of the economy equal to $d=3,4,5$.

We focus first on the case (a) in which $m$ is the only random parameter. The contagion effect depends crucially on the shape of the distribution of $m$. By assumption (a) the distribution $v$ equals a product measure. Letting $\Delta=0.5$, $v$ is thus given by

$$
v(d m, d \Delta)=\chi(d m) \otimes \delta_{0.5}(d \Delta)
$$

In Figures 1, 2, and 3, we plot the approximate loss densities for the interaction cases $d=3,4,5$ for a portfolio of size $r=10000$ choosing

$$
\chi=\delta_{0.5}, \quad \chi=\frac{1}{5} \cdot\left(\delta_{0.3}+\delta_{0.4}+\cdots+\delta_{0.7}\right), \quad \chi=\text { unif }[0.3,0.7]
$$

respectively.

For $\chi=\delta_{0.5}$ (Figure 1) portfolio losses fluctuate around their mean $r \cdot m=$ 5000. For a low degree of connectedness $d$ losses fluctuate more excessively when compared with higher values of $d$. The difference of the contagion effect for varying degrees of interaction $d$ is quite significant in the case in which the support of $\chi$ is small. Observe that in terms of loss probabilities the size of the contagion effect is given by the area between the density curves. 


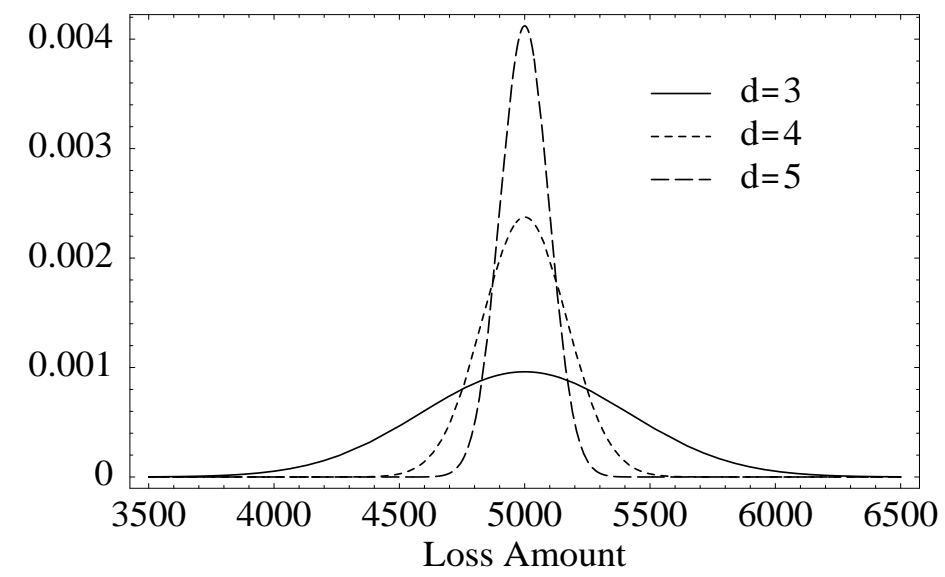

Figure 1: Approximate loss density, varying the degree of connectedness $d\left(r=10.000, \rho=0.5, \Delta=0.5\right.$, and $\left.\chi=\delta_{0.5}\right)$.

In Figure 2 we illustrate the contagion effect for $\chi$ being a convex combination of Dirac measures. In this case strong interaction of firms corresponding to low values of $d$ induces additional fluctuations around the random means $r \cdot \rho=3000,4000, \ldots, 7000$ - leading to a smoother loss density with less prominent peaks. At the same time the tail of the distribution becomes slightly fatter when $d$ decreases, but this effect is less significant than in Figure 1. Observe that the ordinate axis in Figure 2 is differently scaled than in Figure 1. Hence, the size of the contagion effect corresponding to the area between the density curves is indeed considerably smaller.

The approximate loss densities for $\chi=$ unif $[0.3,0.7]$ are shown in Figure 3. For $\chi$ having large support, fat tails due to contagion are not very strong, and the approximate loss distributions do not differ much for various degrees of interaction. We emphasize again that the ordinate axis in Figure 3 is differently scaled than in Figures 1 and 2. The area between the density curves is smallest for $\chi$ being uniform.

The properties of the distribution $\chi$ influences the size of the contagion effect significantly. Let us now look at case (b) investigating the relationship between $\Delta$ and the shape of the losses. It is apparent from (15) that $\Delta$ is a factor in the denominator of the argument of the cumulative normal distribution function. Hence, multiplying $\Delta$ by a constant factor is equivalent to rescaling the difference of the losses from the mean $r \cdot m$ by a constant factor. For both low and high degree of interaction the fluctuations around $r \cdot m$ are multiplied by the same factor. If $\Delta$ is random, loss distributions are simply 


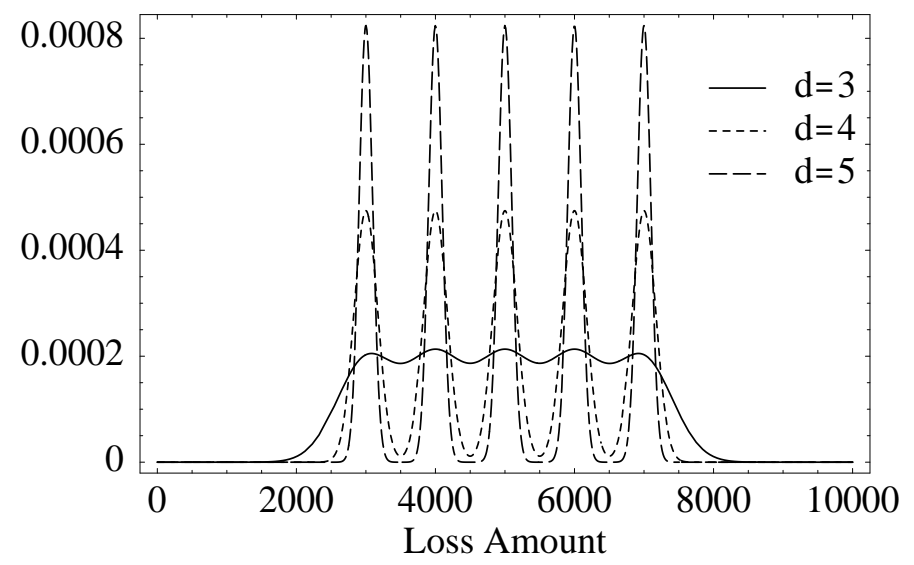

Figure 2: Approximate loss density, varying the degree of connectedness $d(r=10.000, \rho=0.5, \Delta=0.5$, and $\chi=$ $\left.\frac{1}{5} \cdot\left(\delta_{0.3}+\delta_{0.4}+\cdots+\delta_{0.7}\right)\right)$.

superpositions of normal variables with mean $r \cdot m$. Nevertheless, contagion effects are qualitatively the same for non-random and random $\Delta$; i.e. for low degrees of connectedness of the economy fluctuations around the mean $r \cdot m$ are more excessive than for high degrees of connectedness.

If $m$ and $\Delta$ are both random as in case (c), the size of the contagion effect depends on the marginal distribution of $m$. If the marginal distribution of $m$ is close to a uniform distribution, the contagion effect is small. Conversely, if the marginal distribution of $m$ is dominated by peaks, contagion smoothes the loss distribution. If the marginal distribution of $m$ has small support, the contagion effect is strongest. While the contagion effect is determined by the marginal distribution of $m$, the actual shape of the loss distribution for given contagion parameter $d$ is governed by the joint distribution of $m$ and $\Delta$. Observe finally that for given $d$ approximate loss distributions can be very similar for different specifications of the joint distribution of $m$ and $\Delta$; nevertheless, if the marginal distributions of $m$ differ considerably, the size of the induced contagion effects might be quite different when varying $d$.

Let us finally emphasize that the effect of contagion on the distribution of aggregate losses depends critically on the degree $d$ of complexity of the business partner network. Specifically, with increasing complexity $d$ the contagion effect decreases and the tail of the loss distribution becomes lighter. This relation is consistent with the micro-economic bank contagion model of Allen \& Gale (2000), for example; it was recently empirically confirmed by Upper \& Worms 


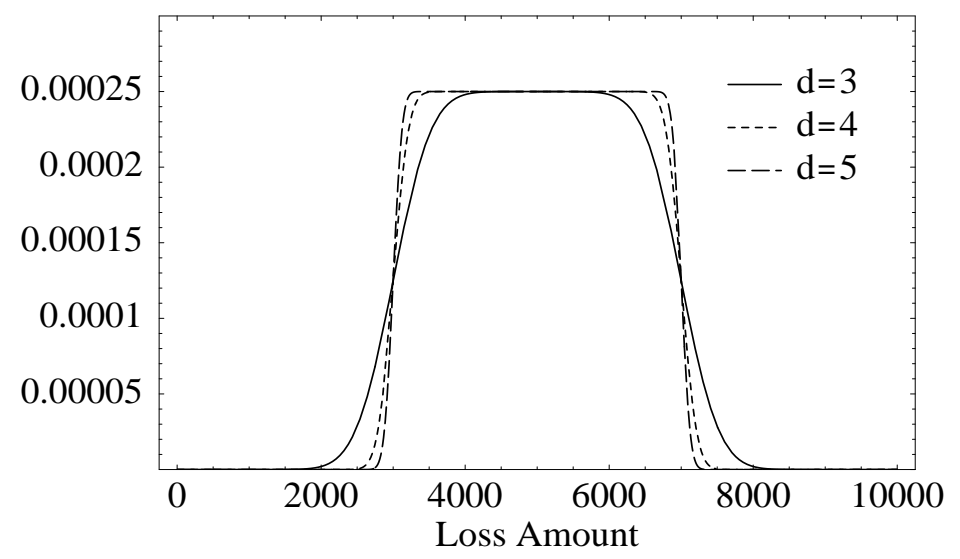

Figure 3: Approximate loss density, varying the degree of connectedness $d(r=10.000, \rho=0.5, \Delta=0.5$, and $\chi=$ unif $[0.3,0.7])$.

(2002). The intuitive idea is that the more complex the connectivity structure of the economy, the lower is the probability of observing persistent and growing clusters of firms in the same liquidity state (see Giesecke \& Weber (2002) for more details).

\section{Bernoulli Mixture Representation}

The class of existing Bernoulli mixture models has proven to be useful in practice to model loss distributions in the context of cyclical correlations between losses on individual portfolio positions. We have argued, however, that the disregard of contagion effects between positions may lead to an underestimation of extreme fluctuation of portfolio losses. In this section we provide a Bernoulli mixture type specification of our model, and thereby enlarge the existing Bernoulli mixture class with a model accommodating both cyclical and contagion effects.

The key to the Bernoulli mixture type representation lies in a particular specification of the conditional distribution $M_{k, x}$ of position losses $U(i)$ for a firm $i \in \Lambda_{n}$ in interaction-based liquidity state $x \in\{0,1\}$ when the state of the macro-economy is $k \in \mathbb{R}^{d}$. We put

$$
M_{k, x}= \begin{cases}1 & \text { with probability } P_{x}(k) \\ 0 & \text { with probability } 1-P_{x}(k)\end{cases}
$$


so that, conditionally on $(k, \xi)$, the position loss is a Bernoulli random variable with parameter $P_{x}(k)$, and we have $\tilde{l}(k)=\left(P_{0}(k), P_{1}(k)\right)$. The probability $P_{x}(k)$ is supposed to depend on $x$ and $k$ in a measurable way. We can interpret $P_{x}(k)$ as the probability of default for a firm in interaction state $x$ when the economy is in state $k$, which results in a loss of one unit of account. Since position losses are conditionally independent given $(k, \xi)$ by assumption, the particular specification (17) leads to our model becoming one of the Bernoulli mixture class.

Given the Bernoulli mixture representation of our model, we can take advantage of the existing estimation models for the mixing distribution. Taking into account the homogeneity of firms in our economy, some of these models are outlined in the examples below.

Example 4.1 (Bernoulli regression model). Let $F: \mathbb{R} \rightarrow[0,1]$ be some strictly increasing continuous function, and let $\alpha_{1}, \alpha_{2}, \alpha_{3}$ be regression parameters with $\alpha_{2}>0$. We let $K \in \mathbb{R}$ be one-dimensional with given distribution and put

$$
P_{x}(K)=F\left(\alpha_{1} K+\alpha_{2} x+\alpha_{3}\right) .
$$

For different choices of the regression relationship we refer to Joe (1997). The one-factor regression model may not be flexible enough; we can generalize to higher dimensions of the macro-factor vector $K$. We start with a choice corresponding to the CreditRisk + model structure.

Example 4.2 (Gamma model). Let $\alpha=\left(\alpha_{1}, \ldots, \alpha_{p}\right) \in \mathbb{R}_{+}^{p}$, and $\gamma_{1}, \gamma_{2}>0$ be factor weights. Let $K \in \mathbb{R}^{p}$ be a p-dimensional iid-Gamma vector and put

$$
P_{x}(K)=1-\exp \left(-\sum_{i=1}^{p} \alpha_{i} K_{i}-\gamma_{1} x-\gamma_{2}\right) .
$$

Example 4.3 (Probit normal model). Let $\alpha=\left(\alpha_{1}, \ldots, \alpha_{p}\right), \gamma_{1}>0$, and $\gamma_{2}$ be factor weights. Let $K \in \mathbb{R}^{p}$ be a p-dimensional normally distributed random vector and put

$$
P_{x}(K)=\Phi\left(-\sum_{i=1}^{p} \alpha_{i} K_{i}+\gamma_{1} x+\gamma_{2}\right) .
$$

This model is closely related to the one exploited in KMV and CreditMetrics. The assumption of normality of the factors is not essential; other distributions such as the $t$-distribution or more general mean-variance mixtures are possible, see Frey \& McNeil (2001). The following specification is similar in spirit to the CreditPortfolioView model. 
Example 4.4 (Logit normal model). Let $\alpha=\left(\alpha_{1}, \ldots, \alpha_{p}\right), \gamma_{1}>0$, and $\gamma_{2}$ be factor weights. Let $K \in \mathbb{R}^{p}$ be a p-dimensional normally distributed random vector and put

$$
P_{x}(K)=\left(1+\exp \left(-\sum_{i=1}^{p} \alpha_{i} K_{i}+\gamma_{1} x-\gamma_{2}\right)\right)^{-1} .
$$

We now investigate the approximate loss distribution (11) for the specification (17) of our general model under various assumptions on the dependence between firms. This allows to evaluate the effects of cyclical default correlation and credit contagion on the fluctuation of aggregate losses in the context of the Bernoulli mixture model class. The results indicate that contagion can indeed be a significant factor, as it can lead to (potentially severe) fluctuations in losses in addition to that related to fluctuations in the macro-environment.

In order to focus on the effects of firm dependence, we assume that $Q=\delta_{\rho}$ : the average proportion (5) of firms in interaction-induced liquidity state 1 is equal to the constant $\rho$. It remains to specify a model for the default probability $P_{x}(K)=l_{x}(K)$ together with the distribution of the macro-factor $K$. We choose a one-factor version of the Probit normal model of Example 4.3, which parallels the models of KMV and CreditMetrics:

$$
P_{x}(k)=\Phi\left(-\alpha k+\gamma_{1} x-\gamma_{2}\right)
$$

We set $\alpha=1, \gamma_{1}=2$, and $\gamma_{2}=3$, considering different choices for the law of the macro-economic factor $K$.

Under our current assumptions, for a portfolio of size $r>0$ the function (11) uniformly approximating the probability of aggregate losses being larger than $a>0$ becomes

$$
\Psi_{d, a}(r)=\int \Phi\left(\frac{\sqrt{r} m\left(\rho, l_{0}(k), l_{1}(k)\right)-a / \sqrt{r}}{\left(l_{1}(k)-l_{0}(k)\right) \sigma(d, \rho) r^{1 / d}}\right) \kappa(d k),
$$

where $l_{x}(k), m$, and $\sigma$ are given by (18), (9), and (10), respectively. For comparison, we shall also study the case where the firms do not interact with their business partners, meaning that contagion effects are not present. In this situation we replace the extremal invariant distribution $\nu_{\rho}$ of firms state in (4) with a product $\pi_{\rho}$ of Bernoulli measures with density $\rho$. For the loss approximation we then obtain

$$
\Psi_{d, a}^{\pi}(r)=\int \Phi\left(\frac{\sqrt{r} m\left(\rho, l_{0}(k), l_{1}(k)\right)-a / \sqrt{r}}{\tilde{\sigma}(k, \rho)}\right) \kappa(d k),
$$




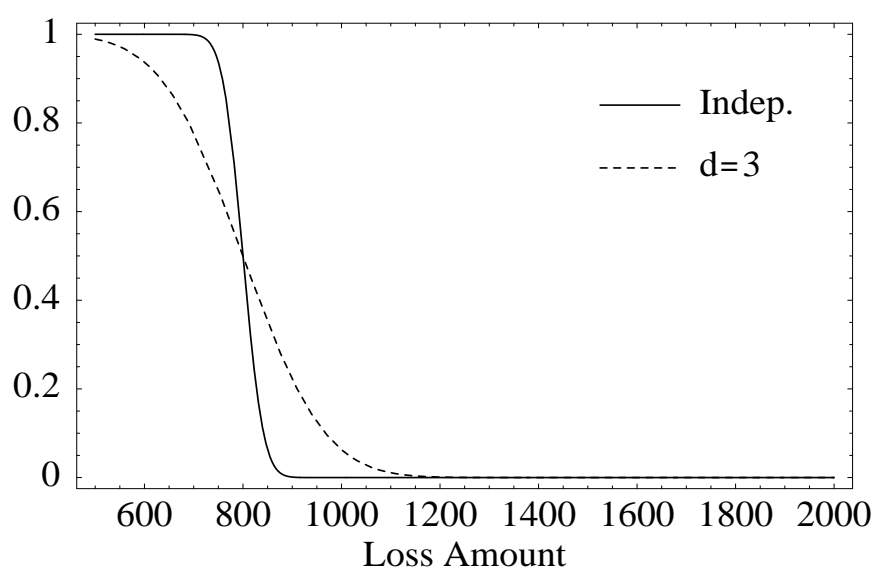

Figure 4: Approximate loss distribution in the one-factor Probit normal model (18) when the macro-parameter $K$ is certain and set equal to zero, for $d=3$ and the independence case $(r=10.000$ and $\rho=0.5)$.

with limiting variance $\tilde{\sigma}^{2}(k, \rho)$ given by

$$
\tilde{\sigma}^{2}(k, \rho)=(1-\rho) \cdot \operatorname{var}\left(M_{k, 0}\right)+\rho \cdot \operatorname{var}\left(M_{k, 1}\right)+\rho(1-\rho) \cdot\left(l_{1}(k)-l_{0}(k)\right)^{2},
$$

where, in the current setup, $\operatorname{var}\left(M_{k, x}\right)=P_{x}(k)\left(1-P_{x}(k)\right)$.

We now consider a portfolio of size $r=10000$, where the probability $\rho$ of an individual firm to be liquidity stressed is equal to $50 \%$. In Figures 4,5 and 6 we plot the approximate loss distribution for different specifications of the law of the macro-parameter $K$. That is, we assume $K$ to be distributed according to

(d) a Dirac measure placing mass one on the value 0 (Fig.4);

(e) a Gaussian distribution with mean 0 and variance 1 (Fig.5); and

(f) a Gaussian distribution with mean -4 and variance 0.05 (Fig.6).

In the figures we compare the case where firms are independent (corresponding to (20)), and where firms do interact in an economy with degree of complexity $d=3$ (corresponding to (11) and (19)). In contrast to Figures 1, 2 and 3 we do not plot densities, but excess probabilities; thus, the size of the contagion effect is measured by the difference between the two functions in Figures 4, 5 and 6 , respectively. 


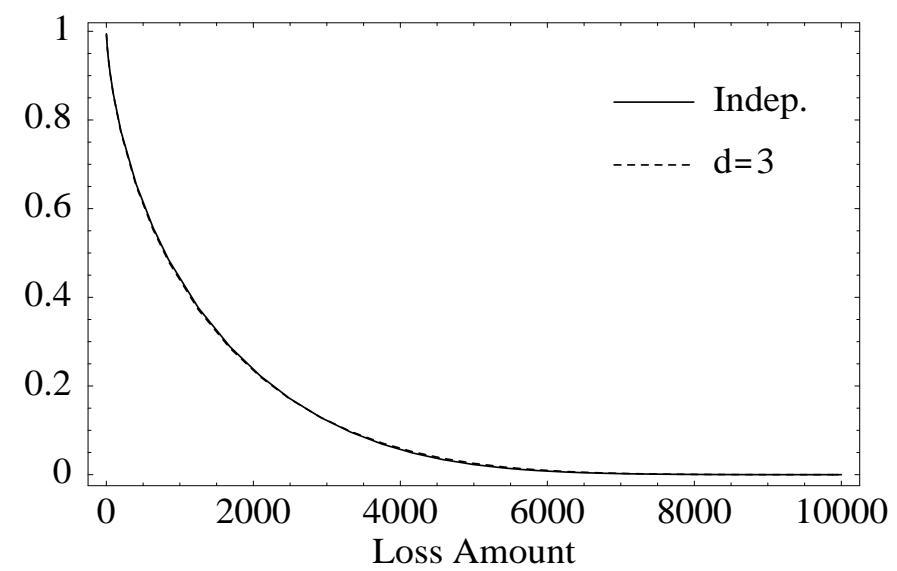

Figure 5: Approximate loss distribution in the one-factor Probit normal model (18) when the macro-parameter $K$ is standard normally distributed, for $d=3$ and the independence case $(r=10.000$ and $\rho=0.5)$.

In cases (d) and (f) a considerable contagion effect is present with fatter tails for contagion-prone than for independent firms (Fig. 4 and 6). In case (e) the difference between the excess loss probabilities has almost disappeared and no significant contagion effect is visible (Fig. 5). The different size of the contagion effects can easily be understood if we re-parameterize the model in terms of the variables $m$ and $\Delta$ given in (12) and (13) and if we recall our discussion of the cases (a), (b) and (c) from Section 3.2. The contagion effect is governed by the marginal distribution of $m$ which can be derived from Figure 7. In the upper half of Figure 7 the variables $m$ and $\Delta$ are shown as a function of $k$ for the one-factor Probit normal model (18) and the given parameter values. The lower half displays the densities of the Gaussian distributions of factor $K$ in the case (e) and (f), respectively.

Due to the low variance of the Gaussian factor distribution in case (f) $K$ takes values close to its expectation with very high probability. Close to the expected value -4 of the factor $K$, the slope of $k \mapsto m(k)$ is not very large. Hence, the law of $m$ is close to a Dirac measure. Consistent with our discussion in Section 3.2 we observe in Figure 6 a significant contagion effect. Figure 7 shows that for low values of $k$ the variable $m$ takes values close to one corresponding to a bad macro-environment. Conversely, in case (d) $K$ is deterministic and equal to 0 giving rise to a low deterministic value for $m$ which corresponds to a good macro-economic environment. As expected, comparison 


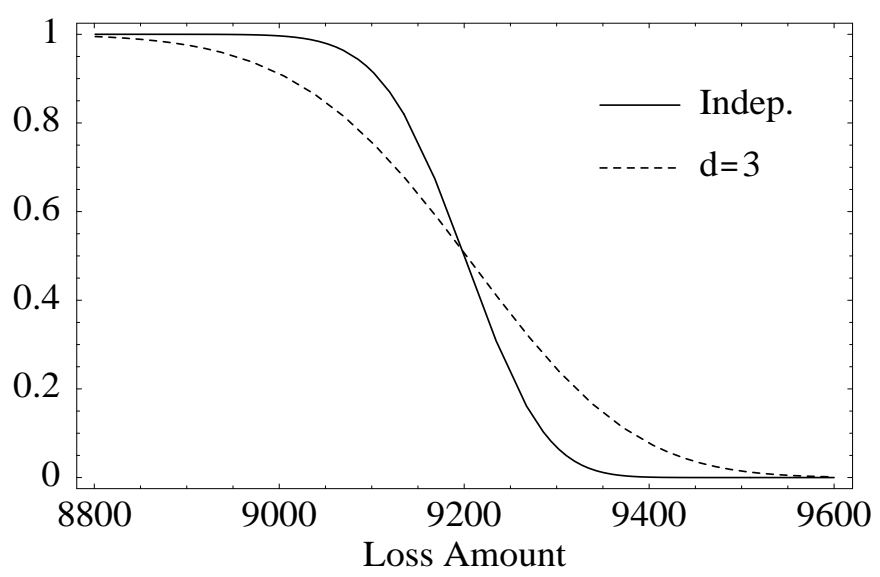

Figure 6: Approximate loss distribution in the one-factor Probit normal model (18) when the macro-parameter $K$ is normally distributed with mean -4 and variance 0.05 , for $d=3$ and the independence case $(r=10.000$ and $\rho=0.5)$.

of Figures 6 and 4 shows considerably higher losses in case (f) than in case (d). Let us now investigate the cases (d) and (e) in which the macro-economic factor $K$ has the same expectation 0 but different variance. If $K$ is deterministic, $m$ is deterministic. By our discussion from Section 3.2 we expect a visible contagion effect as confirmed by Figure 4. In contrast, if the law of $K$ is a centered Gaussian with large variance, Figure 7 shows that the distribution of $m$ is atomless placing considerable mass on an interval of significant size. This property of the law of $m$ corresponds to a small contagion effect (Fig. 5) as we have already noticed in the case of a uniform distribution of $m$ in our discussion in Section 3.2 (see also Figure 3).

\section{On Estimating the Model}

In the last section we compared properties of the loss distribution for different model specifications and degrees of connectedness of the economy. In this section we outline the estimation of the model from historical data.

We suppose we are given a sufficiently large set of historical default and loss data. Possible sources include the default and recovery data frequently published by the public credit rating agencies, such as Moody's or Standard \& Poor's, as well as data collected internally in financial institutions on proprietary portfolio positions. In a first step we discriminate the entities in the 


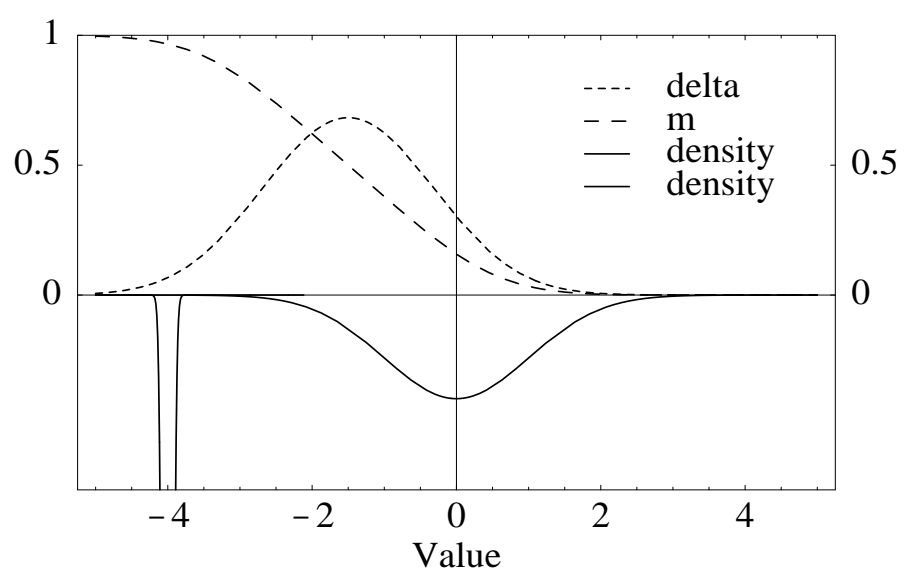

Figure 7: Upper half: Expected loss difference $\Delta(k)$ and average loss in the whole economy $m(k)$ as functions of the macroparameter $k$. Lower half: Densities of Gaussian variables with parameters $(-4,0.05)$ and $(0,1)$.

data in "liquidity-stressed" and "liquidity-stable" firms, which correspond to the states $x \in\{0,1\}$. For this we can use, for example, the external or internal credit rating of a firm, or balance-sheet and cash-flow data if available.

The next step consists of choosing the conditional distribution $M_{k, x}$ of position losses on a firm in liquidity state $x \in\{0,1\}$ when the state of the economy equals $k \in \mathbb{R}^{p}$. Because of its practical relevance, we will consider the generalized Bernoulli mixture specification (17), i.e. we put

$$
M_{k, x}= \begin{cases}1 & \text { with probability } P_{x}(k), \\ 0 & \text { with probability } 1-P_{x}(k) .\end{cases}
$$

$P_{x}(k)$ can be interpreted as the probability of default for a firm in liquidity state $x$ when the economy is in state $k$. Following a parametric estimation strategy, we will fix some parametric model for $P_{x}(k)$ together with a distribution for the macro-factors $k$. Standard industry-examples as discussed in Section 4 include the Gamma model, the Probit model, and the Logit model, together with the appropriate factor distribution $\kappa$.

Note that in contrast to standard models which neglect contagion, we separated the data into two pools depending on the liquidity state of the individual firms. In particular, from this the empirical distribution of the default probabilities conditional on the state $x \in\{0,1\}$ of the firms can be obtained. Hence, we can estimate the parametric models $P_{0}(K)$ and $P_{1}(K)$, respectively. In contrast to standard industry practice, in a contagion-based approach $P_{0}(K)$ 
and $P_{1}(K)$ must be estimated under the restriction that in both cases the same parameters are chosen.

In a next step, we estimate the distribution $Q$ of the average number of liquidity-stressed firms $\rho$. For each point in time, the average number of liquidity-stressed firms can be calculated from the data, allowing to estimate $Q$.

Taking the parameter $d$ as given, we are now in a position to calculate approximate loss distributions for large portfolios from (11). With the generalized Bernoulli specification, we have in fact that the approximate probability of aggregate losses exceeding $a>0$ for a portfolio of size $r \in \mathbb{R}_{+}$is given by

$$
\Psi_{d, a}(r)=\iint \Phi\left(\frac{\sqrt{r}\left(\rho P_{1}(k)+(1-\rho) P_{0}(k)\right)-a / \sqrt{r}}{\left(P_{1}(k)-P_{0}(k)\right) \sigma(d, \rho) r^{1 / d}}\right) Q(d \rho) \kappa(d k),
$$

where $\sigma(d, \rho)$ is given by (10). Heuristically, by interpolation between various degrees of interaction we may and will actually assume that $d$ is not necessarily a natural number, but can take on any real value larger than 2 .

The parameter $d$ stands for the degree of complexity of the business partner network; as discussed in Section 3 it governs the size of the contagion effect present in the economy. Given a homogeneous portfolio of firms, we need to determine its degree of connectedness $d$ if we wish to calculate its loss distribution. To do so, we introduce contagion indicators and contagion rating classes $C=\left\{c_{1}, \ldots, c_{m}\right\}$. Contagion indicators can for example be the number of business partner relations or the number of trade credit relationships an individual firm possesses on average. We will assume that we can assign a contagion rating to a homogeneous portfolio via the indicators.

With every contagion rating class $c \in C$ we will associate a contagion parameter $d$ using our historical data. For a contagion class $c \in C$, the historical loss distribution can estimated. Comparison with the loss distribution generated by the model for various degrees of connectedness $d$ allows us to estimate the contagion parameter $d$ related to any rating class in $C$. We emphasize that in the choice of $d$ we can allow for real numbers larger than 2 .

Finally, suppose we have estimated the model and we are interested in predicting the loss distribution for some given actual credit portfolio. The contagion indicators can be used to obtain the contagion rating of the portfolio, which in turn corresponds to a contagion parameter $d$ that was obtained by our calibration procedure. By (22), we can now calculate the approximate loss distribution of the portfolio. 


\section{Conclusion}

A thorough understanding of aggregate credit loss risk associated with large portfolios of financial positions is of critical importance for the management of financial institutions and the regulatory authorities supervising financial markets. In aggregating individual risk exposures the dependence between losses on positions is the most significant factor. In that respect the standard Bernoulli mixture models widely applied in the financial industry focus exclusively on cyclical correlations between firms' positions, which are due to the dependence of firms on the common macro-environment. Because of its ignorance of the empirically well-documented default contagion processes [see Schoenmaker (2000), for example], such an approach typically underestimates aggregate loss risks. In response to that, in this paper we model the local interaction of firms with their business partners and the associated contagion processes, in addition to cyclical correlation effects. We explicitly approximate the distribution of aggregate credit losses on large financial portfolios.

With the loss distribution at hand we are able to quantify the relation between the variability of global (macro-) economic fundamentals, strength of local interaction between firms, and the fluctuation of portfolio losses, i.e. the degree of aggregated credit loss risk. We find that cyclical oscillations in economic fundamentals dominate average portfolio losses, while local firm interaction and the associated contagion processes cause additional fluctuations of losses around their average. These additional fluctuations correspond to additional loss risks, which are not due to the cyclical dependence between firms. As recently confirmed by empirical studies, the strength of the contagioninduced loss variability and hence the tail properties of the loss distribution depends on the degree of complexity of the business partner network, i.e. the degree of connectedness between firms in the economy.

For regulatory authorities our results have significant implications. First, credit contagion phenomena cause typically additional loss risks, which are not accounted for by the standard industry models. The potential underestimation of total credit loss risk can lead to capital provisions which may prove to be insufficient to buffer actual losses. Second, the effects of credit contagion are less severe in an economy in which firms operate within a complexly structured business partner network. Regulatory policy supporting complexity and diversity in business relations among firms thus helps to mitigate adverse credit contagion effects and reduce the degree of systemic risk in the financial market. 


\section{A Normal Approximation}

Proof of Theorem 3.1. Assume first that $Q=\delta_{\rho}$ and $\kappa=\delta_{k}$ for $\rho \in(0,1)$ and $k \in \mathbb{R}_{+}$. In this case, the approximation reduces to the case of Theorem 4.4. of Giesecke \& Weber (2002), and we get that

$$
\sup _{a \in \mathbb{R}}\left|\beta\left(L_{n} \geq a\right)-\Psi_{d, a}\left(\left|\Lambda_{n}\right| ; \rho, \tilde{l}(k)\right)\right| \leq \epsilon_{n},
$$

where $\epsilon_{n} \rightarrow 0$ as $n \rightarrow \infty$.

For given $k$ and $\rho$, the distribution of

$$
\left|\Lambda_{n}\right|^{-\frac{d+2}{2 d}}\left(L_{n}-\left|\Lambda_{n}\right| \cdot m(\rho, k)\right)
$$

under the measure

$$
\int\left(\otimes_{i \in \mathbb{Z}^{d}} M_{k, \xi(i)}\right)(d w) \nu_{\rho}(d \xi), \quad w \in \mathbb{R}_{+}^{\mathbb{Z}^{d}}
$$

will be denoted by $\varsigma_{\rho, k}^{n}$. We define the quantity

$$
\delta_{\rho, k}^{n}:=\sup _{n^{\prime} \geq n} \sup _{z \in \mathbb{R}}\left|\varsigma_{\rho, k}^{n^{\prime}}([z, \infty))-\Phi\left(-\frac{z}{\left(l_{1}(k)-l_{0}(k)\right) \cdot \sigma(\rho)}\right)\right|,
$$

where $\Phi$ is the Gaussian distribution function.

Inequality (23) implies that $\delta_{\rho, k}^{n}$ converges to 0 for all $\rho \in(0,1)$ and $k \in \mathbb{R}^{p}$ as $n \rightarrow \infty$. Observe that $(\rho, k) \mapsto \delta_{\rho, k}^{n}$ is measurable. For $\epsilon>0$ we can therefore define measurable sets

$$
A_{\epsilon}^{n}=\left\{(\rho, k) \in(0,1) \times \mathbb{R}^{p}: \delta_{\rho, k}^{n}<\epsilon\right\} .
$$

Then $A_{\epsilon}^{n} \subseteq A_{\epsilon}^{n+1}$, and $(Q \otimes \kappa)\left(A_{\epsilon}^{n}\right) \nearrow 1$ as $n \rightarrow \infty$. Choose $n_{0}$ large enough such that

$$
(Q \otimes \kappa)\left(A_{\epsilon}^{n_{0}}\right) \geq 1-\epsilon
$$

Let $(\rho, k) \mapsto z(\rho, k)$ be a measurable mapping. Then for all $n \geq n_{0}$ we get

$$
\begin{aligned}
& \left|\int\left[\varsigma_{\rho, k}^{n}([z(\rho, k), \infty))-\Phi\left(-\frac{z(\rho, k)}{\left(l_{1}(k)-l_{0}(k)\right) \sigma(\rho)}\right)\right] Q(d \rho) \kappa(d k)\right| \\
\leq & 2\left(1-(Q \otimes \kappa)\left(A_{\epsilon}^{n}\right)\right) \\
& \quad+\sup _{(\rho, k) \in A_{\epsilon}^{n}} \sup _{z^{\prime} \in \mathbb{R}}\left|\varsigma_{\rho, k}^{n}\left(\left[z^{\prime}, \infty\right)\right)-\Phi\left(-\frac{z^{\prime}}{\left(l_{1}(k)-l_{0}(k)\right) \sigma(\rho)}\right)\right| \\
\leq & 3 \epsilon
\end{aligned}
$$


Let $a \in \mathbb{R}$ be arbitrary, and let $n \geq n_{0}$. We can choose

$$
z(\rho, k)=\left|\Lambda_{n}\right|^{-\frac{d+2}{2 d}}\left(a-\left|\Lambda_{n}\right| m(\rho, k)\right) .
$$

It follows that for any $a \in \mathbb{R}$ and $n \geq n_{0}$ the following inequality holds

$$
\begin{aligned}
& \mid \iint \nu_{\rho}\left(L_{n} \geq a\right) Q(d \rho) \kappa(d k) \\
& \quad-\quad \iint \Phi\left(\frac{\left|\Lambda_{n}\right|^{1 / 2} m(\rho, k)-\left|\Lambda_{n}\right|^{-1 / 2} a}{\sigma(\rho)\left|\Lambda_{n}\right|^{1 / d}}\right) Q(d \rho) \kappa(d k) \mid \leq 3 \epsilon .
\end{aligned}
$$

\section{References}

Allen, Franklin \& Douglas Gale (2000), 'Financial contagion', Journal of Political Economy 108, 1-33.

Crouhy, Michel, Dan Galai \& Robert Mark (2000), 'A comparative analysis of current credit risk models', Journal of Banking and Finance 24, 59-117.

CSFP (1997), CreditRisk+: A credit risk management framework. Technical Document, London.

Frey, Rüdiger \& Alexander J. McNeil (2001), Modeling dependent defaults.

Working Paper, Department of Mathematics, ETH Zürich.

Giesecke, Kay \& Stefan Weber (2002), Credit contagion and aggregate losses. Working Paper, Cornell University and Technische Universität Berlin.

Gupton, Gregory, Christopher Finger \& M. Bhatia (1997), CreditMetrics. Technical Document, New York.

Joe, Harry (1997), Multivariate Models and Dependence Concepts, Chapman and Hall, London.

Kealhofer, Stephen (1998), Portfolio management of default risk. Working Paper, KMV Corp.

Keenan, Sean (2000), Historical default rates of corporate bond issuers 19201999. Moody's Investors Services.

Kiyotaki, Nobuhiro \& John Moore (1997), Credit chains. Working Paper, London School of Economics. 
Kondo, Kei-ichi \& Takashi Hara (1987), 'Critical exponent of susceptibility for a class of general ferromagnets in $d>4$ dimensions', Journal of Mathematical Physics 28(5), 1206-1208.

Lang, Larry \& Rene Stulz (1992), 'Contagion and competitive intra-industry effects of bankruptcy announcements', Journal of Financial Economics $32,45-60$.

Liggett, Thomas (1985), Interacting Particle Systems, Springer-Verlag, Berlin.

Liggett, Thomas (1999), Stochastic Interacting Systems: Contact, Voter, and Exclusion Processes, Springer-Verlag, Berlin.

Schoenmaker, Dirk (2000), Contagion risk in banking. Working Paper, Ministry of Finance, the Netherlands.

Upper, Christian \& Andreas Worms (2002), Estimating bilateral exposures in the german interbank market: Is there a danger of contagion? Working Paper, Deutsche Bundesbank.

Wilson, Thomas C. (1997), 'Portfolio credit risk (i)', Risk 10(9), 111-117. 